\title{
Bramatirtye Sortifted.
}

\section{Gimleifung.}

\section{Antma num BamljeIm.}

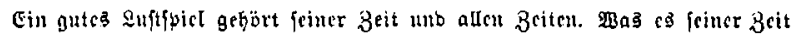

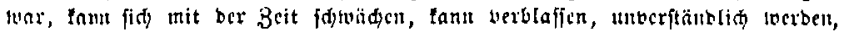

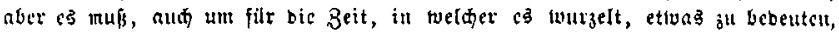

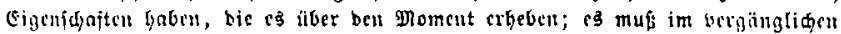

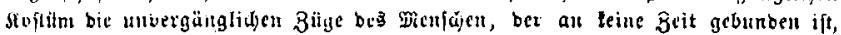

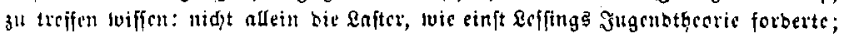

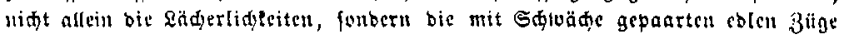
Dis Mlenfiden. Destn trie fein Mlenfd blop lafterhaft ober läderlid ift, gicbt es

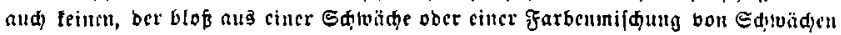

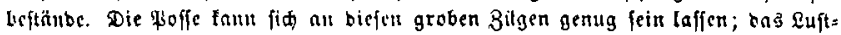

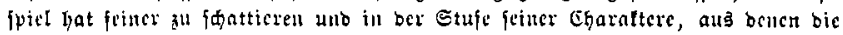

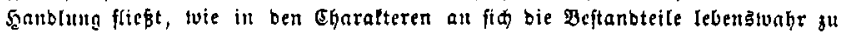
vereinigen, unbelünmert barum, ob es̆ lvirlti

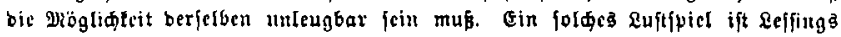

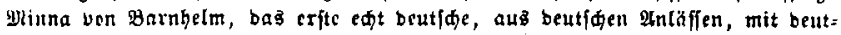

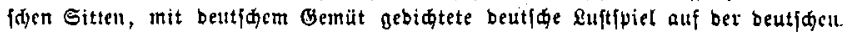

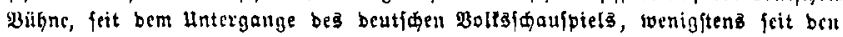

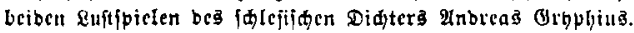

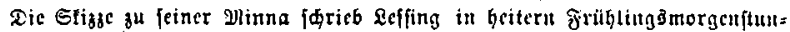

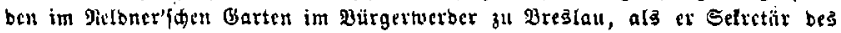
Oencrals Tauengien war, urb arbeitete biefelbc aus, ats er naథ Berlill zurid =

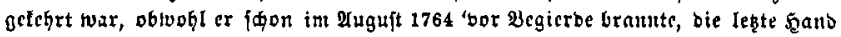

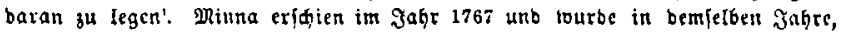
Haфbem fic anfangs ouf preufific Beranlafimg beanjtanbet ober verboten war,

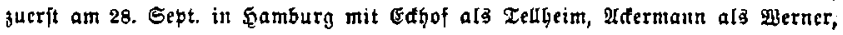

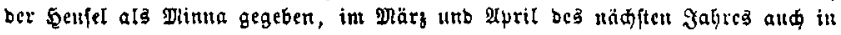
Berlin zebrinal umunterbroden bor cinem voucn \$aufe. Wo hätte bas ङtüd aud freubiger aufgenommen werber follen, als in ber Gauptftabt preufens, ein Stild, bas zur Berberrfidung bes Solbatenftanbes gebiditet war? 


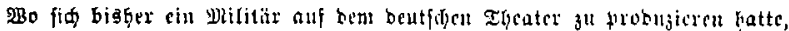

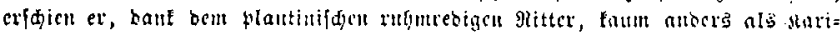

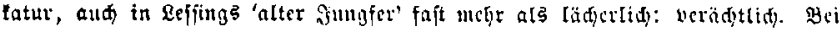
jeincm folbatijacn Dertehr batte ¿cfïing ben Stanb, Dffizicre wir Sorbaten, aud,

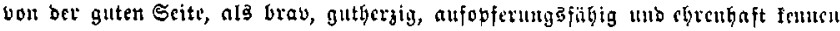

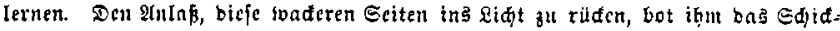

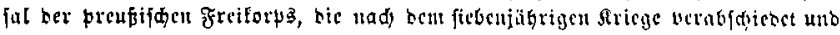

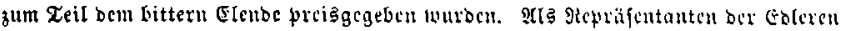

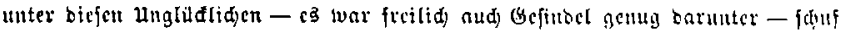

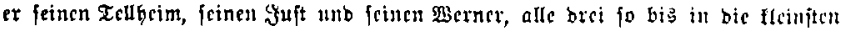

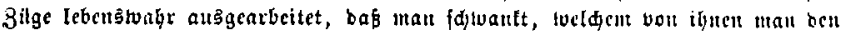

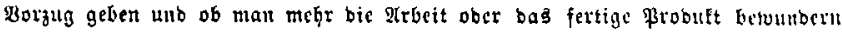
folf. Daneben ben für feinen Berbienft Seforgten, aber barüber binalts auth zu

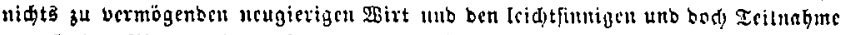
crwedcuben Miccaut, ber crit aun Mtitleib bcluegt, mit guter Irt nimmt unb fid

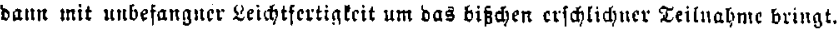

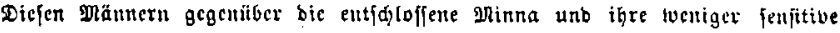

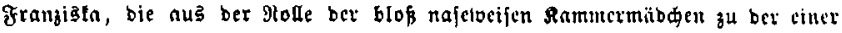

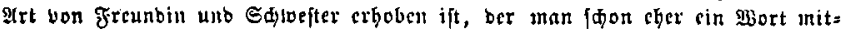
jufpredien geftattet. Die Fabel bes Stüđz, oblvohl fie vortrefflide lomifde utb rügrenbe Eituntionen ansege bringt, ift gegen bie charaftere cin svenig fojluad

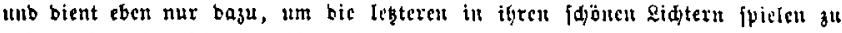

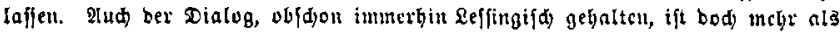

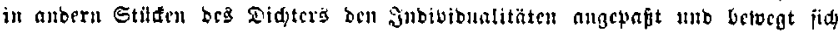

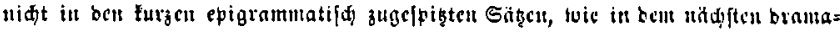
tifdeu Gräeugniffe Refíngg.

Das Ruftfpiel lourbe, natürlid mit ben exforberlidjen "\$orbefferungen" ing

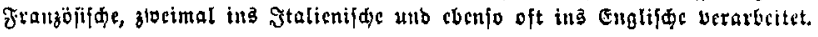

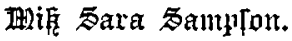

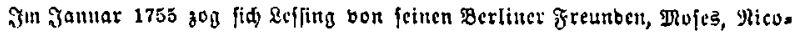
Ini uns PFamler autild, un in Potsban fi申 ber 2uşarbcitung cimes Zraucripicl= cutsurfó ungeföbt. Juibmen zu tönen. Es war פhís Sara Sampion, mit ber ar

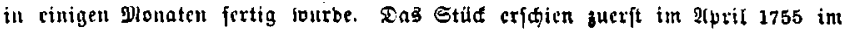

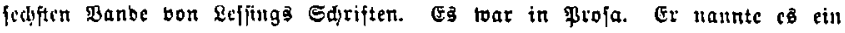

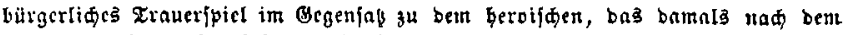
Dhufter ber Frangofen faft ausfthließenb auf ber beutfकen \$ühne galt. Einige

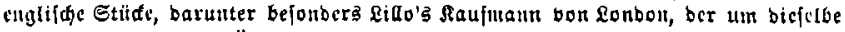

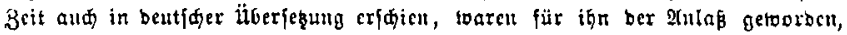
bem Ocbanten tweiter nadinugehen, ob bent bas Sdjidfal, bas, nadj feiter ba= maligen Jntcrpretation bes 2 riffoteles, MitIcib unb Sđreden erregen joll, auf bie Filtrftenbäufer befdräntt, ober au由 in Rreifen, bie uns näber liegen, vorbanbelt fci. Die frage glaubte cr bejafert gu milffen, ba bas, was uns im Sdjiffal ber

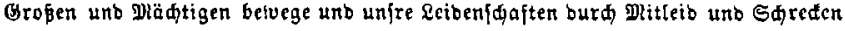

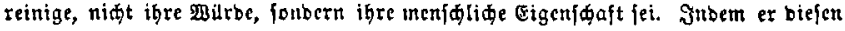
Gebanten in eittem mittleren, zivifden filrft unb Biirger liegenben sreife zu ver=

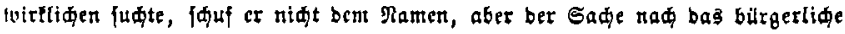




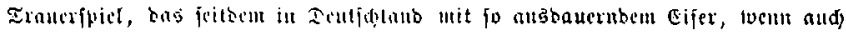

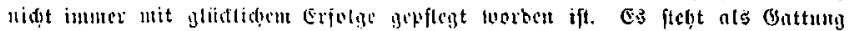

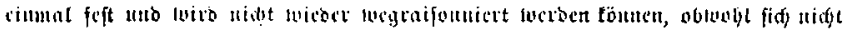

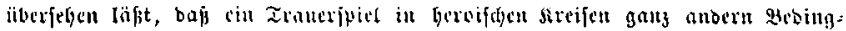

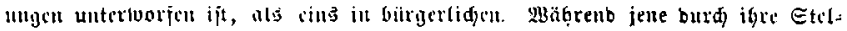

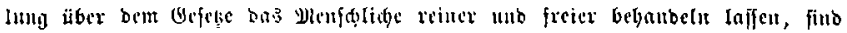

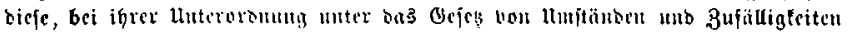

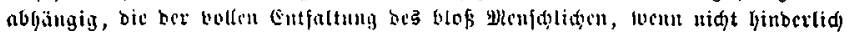

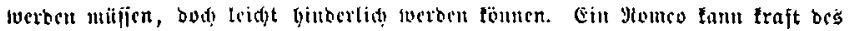

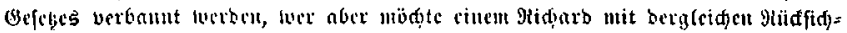

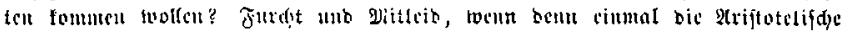

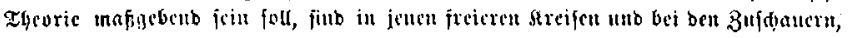

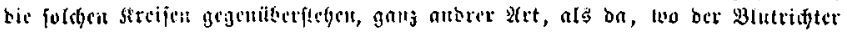

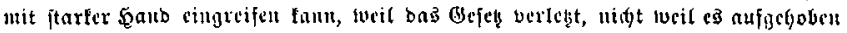

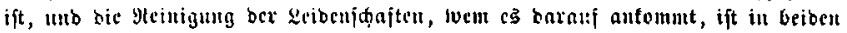
Fölleu werfdicbuter 2 (t)t.

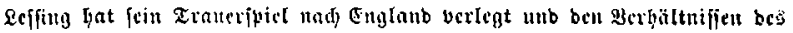

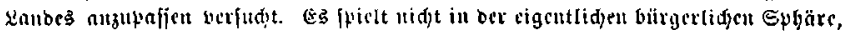

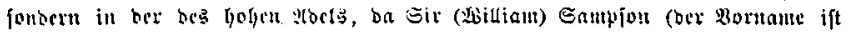
crft 1771 in ber torrigietten Enra binjugejïgt) ber Bater bor Titelgetbin ift. Sill

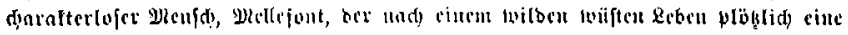

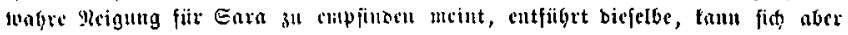

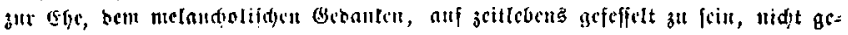

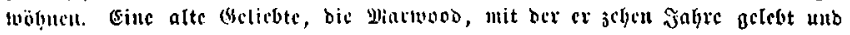

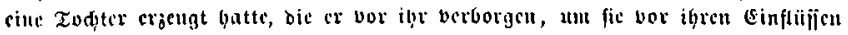

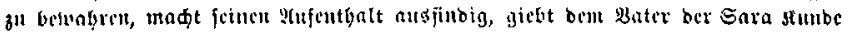

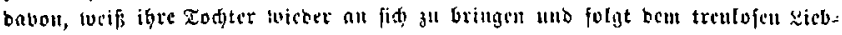

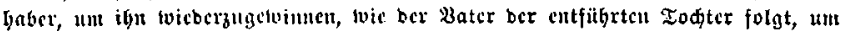

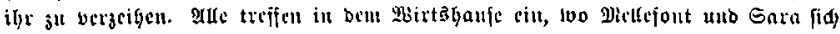

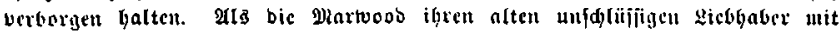

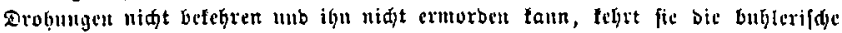

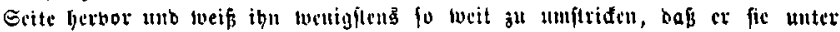
frembcm Namen mit feiner Snta zufammenbringt umb ihs fo, als ex cinmal burdy

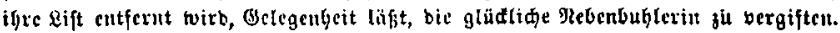
Dic fterbente Eara, bie bas ganze Stiid hiuburd fo fehr nad Bergeigung ge=

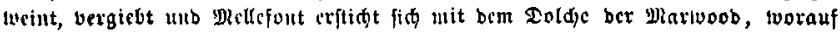

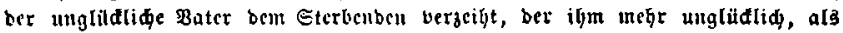

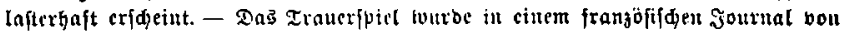

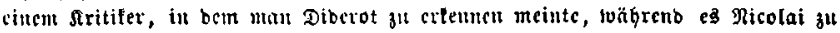
feill fdeiut, fehr gelobt, olyue baß̧ bic wlïngel in ber Anlage ber Fabel verfaunt

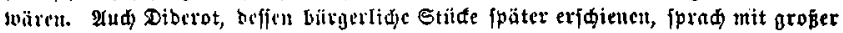

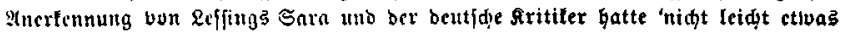
fo Mührenbes gelefen, als bicfo glüdlide Erbidtung, fo uns mit Sd)auber unb

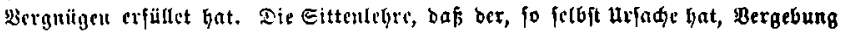
ॐแ Jü̈nfden, vergeben foll, ift unbermerft cingebradt, unb in eincm fefg ftarlen gidite, ba wo mant fic nibt arluartet, vorgefteflet'. 


\section{(rimilia (bitalnffi.}

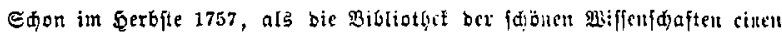

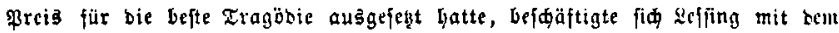

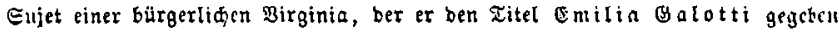
batte. Er arbeitete fehr langfam, 'alle fieben Tage ftebelt Zeilen'. Was er batoul fartig bradte, bermarf or in ber folge wieber unb lehrte erfit zegh Jahre fpätcr,

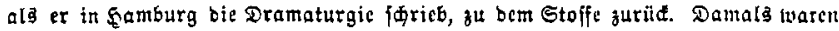

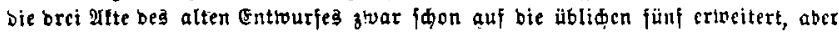
bie 2lrbcit toar jo angelegt, baß fie nur gefpielt, rie gebrudt tocrben follte. Es war nod teine Drfina ill bem Etüde.

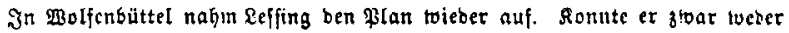
bas alte Eujet, tow bie Frmburger 2lusarbeitung gebrauden, fo loiro bod bic Whlage im allgemeinen geblieben feit, bie 2 uflöfung bes Toditermorbes burd sen Bater ir mobernere Berfältuiffe, abgelöft von bem politifacen Betwert, bas bei ber freien Erfitbung rur ftörenb ober hemmenb inirlent founte. Erft in bicfe bc=

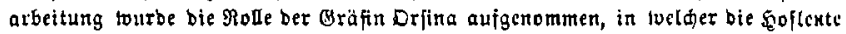
in Braunfdteig, bie Reffing nidt toohl wollten, Bilge ber פarquife Branconi zu erfenten meinten, ehe fie bas Trauerfyiel lenten connter. Denn seffing liés cs bor ber 2luffübrung brufen, unb ans bem Theaternanuftript in Döboslins gefin verlauten modte, fornte nur ungentu feir. Hm bem Gerebe ein Enbc bu

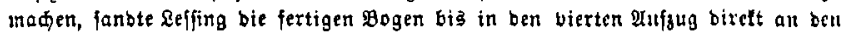
alten $\mathfrak{E} e r z o g$, unb erbot fid, falls berfelbe bie Aluffilfung biefes Traueripicl zum Beburtatage ber Eerzogin für unldidtid) halte, biefelbe unter irgent cincun Iri⿻t fu finbenben Bortanbe zu hintertreiben, ba er nimt geru bas Geringfte thun ober gefdeben laffen wolle, bon bem ber focrog wilnfden tönne, baf sz gar

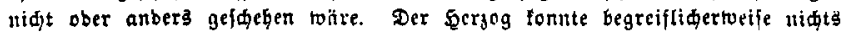

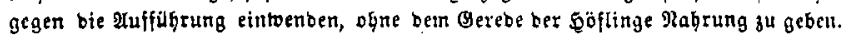

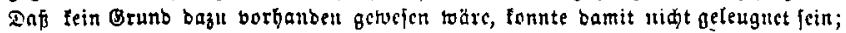

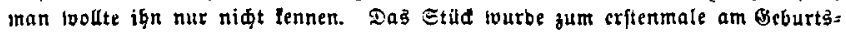

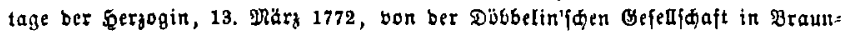
fưlweig aufgefiltert unb bie Darftellung foll zu Döbseling beften gebört babon. ecfing tobnte locber biejer, now ben ral nati einanber folgenben sieberholungen

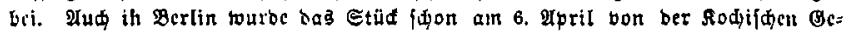
jolf

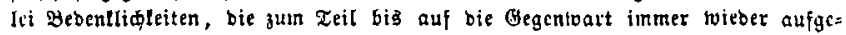
lvorfen werben. 2ber bas Stuid ift geloaltiger getvefen, als alfe Eintourfe bagegen, unb hat fie alle überbauert. Ja in ber neueren Beit hat vielleidt megr beim \$ublifum bon ber Bübne getwirt, als bci scjifing Beitgenofien, bic bei aller

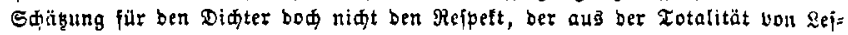
fings Leiftungen entfpringt, auf bas eingelre Etild übertrugen unb, anitatt es gelten ju Iaffen unb baran za Iernen, wo fie nidht betounbern fouten, aus biefent ober jenem theorctifden Sbftem baran zu tabelr fanben. Bwoi Bunltc find unleug=

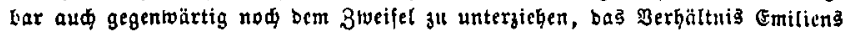

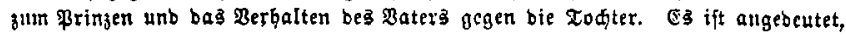

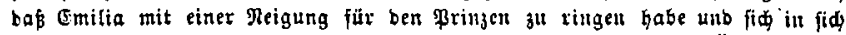

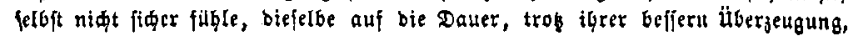




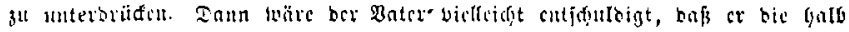

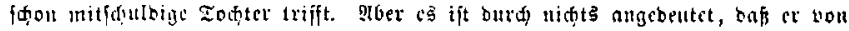

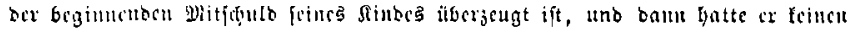

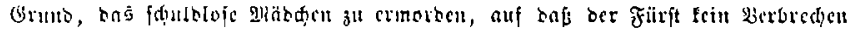

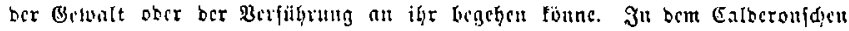

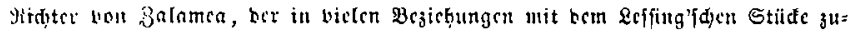

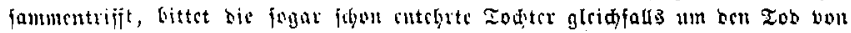

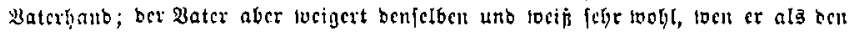

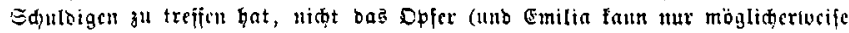

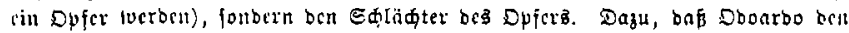

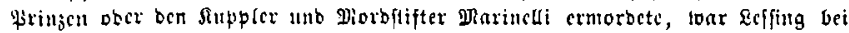

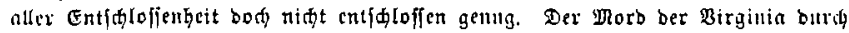

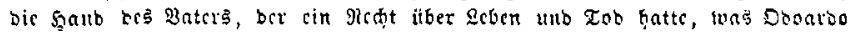

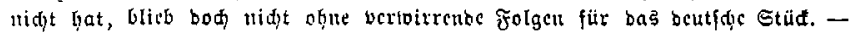

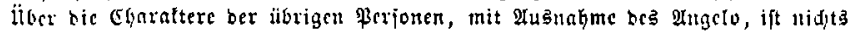
zu fagel, nls boß fie, je genauer fie ftubicht lverben, befto möglider fid betuähren. - Der föruige Dialog hat immer Beluunber gefunben. BBic follte er aud uidgt,

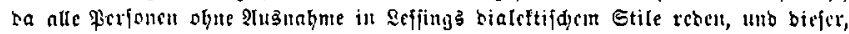
wotn ald uidft ber abjolute Etil, bot. ciul burdans iltbivibuefl voltentecter iff.

\section{Hafhan Der Weite.}

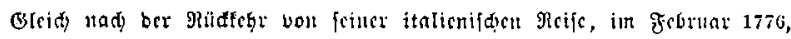
nalym fich \&efiing bor, cinte alten Entwurf zu cinem Edianfpielc, bas ar alf bie

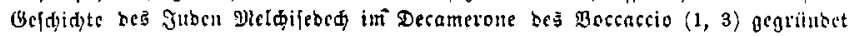

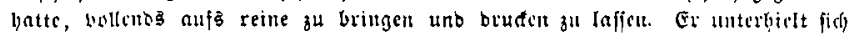

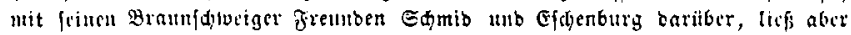

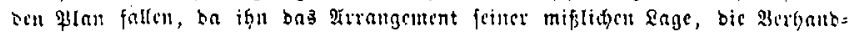

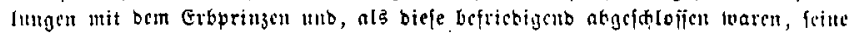
bevorftebente Berbeiratung jerftrcuter. gria sx, nab bem Tobe bex frau, fith in

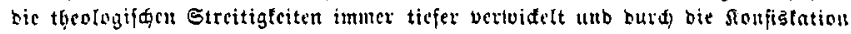

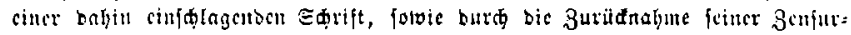

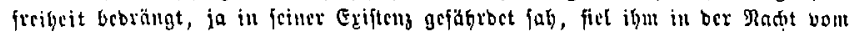
10. auf ben 11. Pluguft 1778 ber alte \$lant wieber cin, an bent er nur cinige ftcine Beränberungen borzutichmen brauble, un feincu Begucrn auf ciure aubern Scite

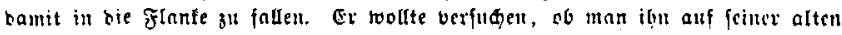
sanjel, alf ben Theater wonigftens nod ungeftöt wolle probigen laffen. Dis

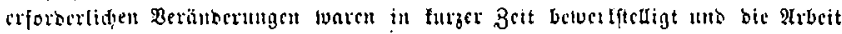

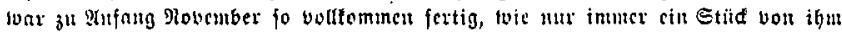

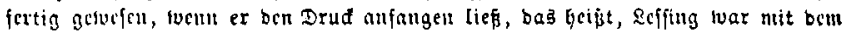

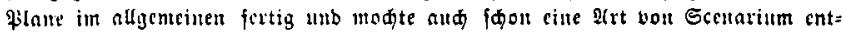

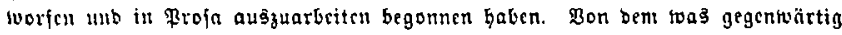

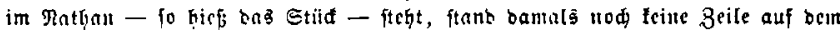

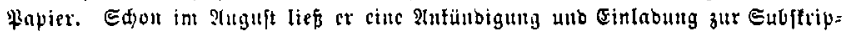

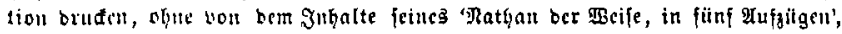

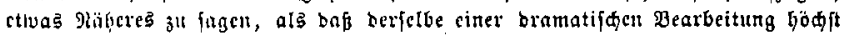

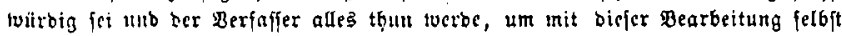

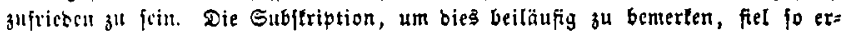




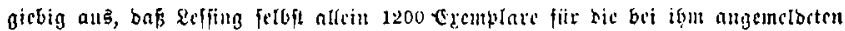
Eubftribenten beburfte. llub bod wufte niemanb, was er zu ervarten habe;

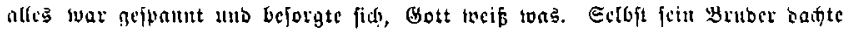

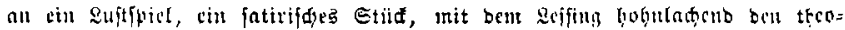

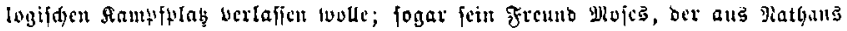

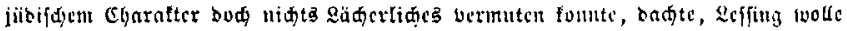

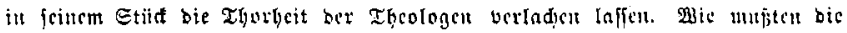

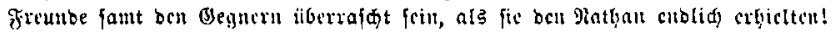

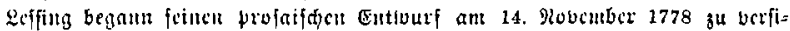

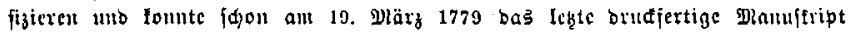

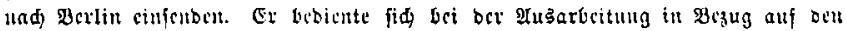

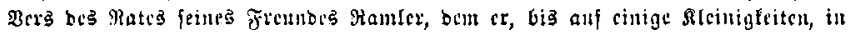

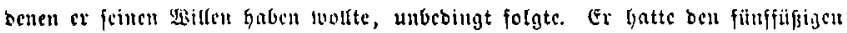

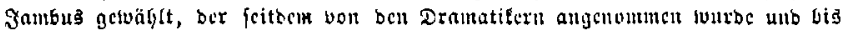

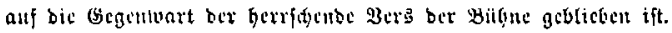

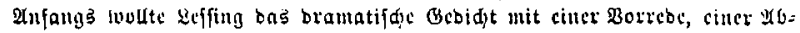

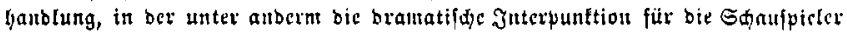

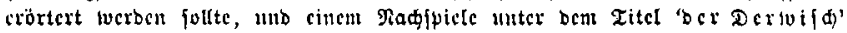

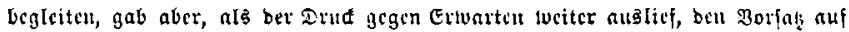

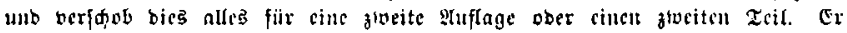
glaubtc im Slauf: bis Goumers 3cit gemug Daju zu yaben, wurbe jebsd surd

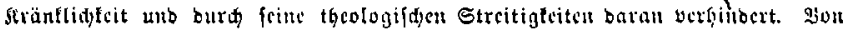

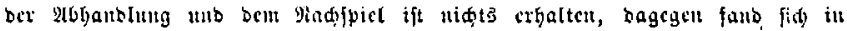

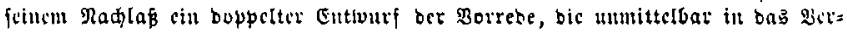

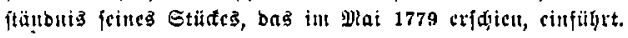

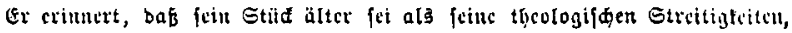

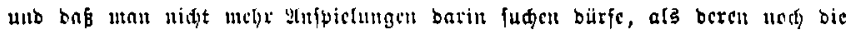

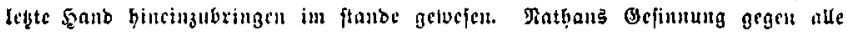

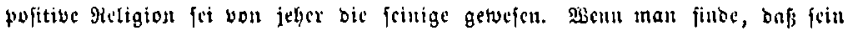
Jiatlyan [chce, cs fgabe von jeber unter allerlei boll scutc gegebel, bic fid

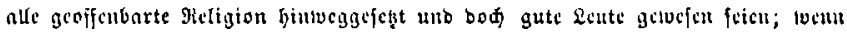

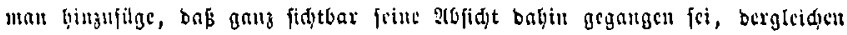

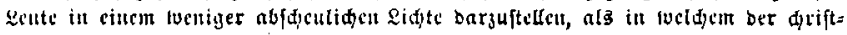

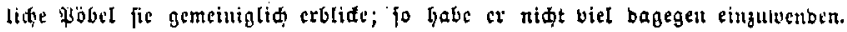

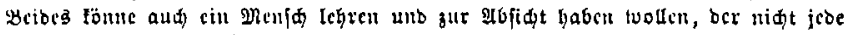

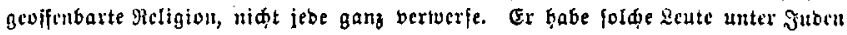

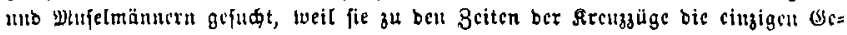

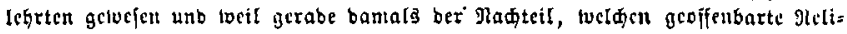

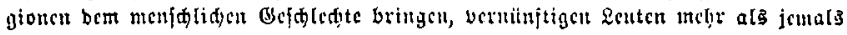

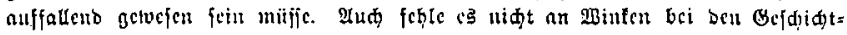
jdjribert, bas citt folder vernilrftiger Maut fid in ciutem Eultan gefulben babs.

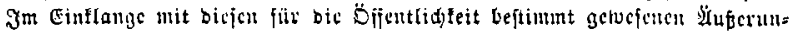

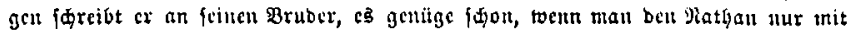
Sntereffe Iefen werbe und unter taufent defen nur Einer baraus an ber Evibedr unt alfgemeinfyeit feincr Ficligion zlocifuth lerne.

ES ift wafyr, eefings Befimung gegen alle pofitive Religion lvar älter als feime tfeologifकen Streitigfeiten infolge but Fraginente. Bentr aud bas Dicifnes 


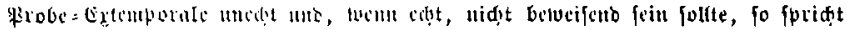

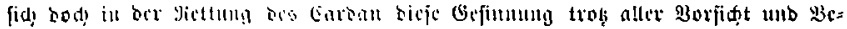

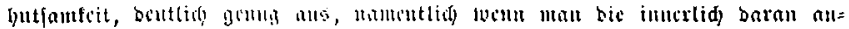

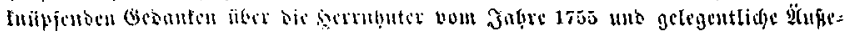

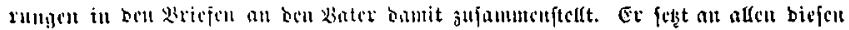

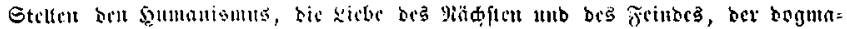

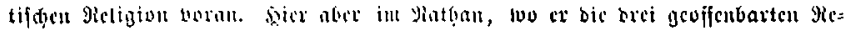

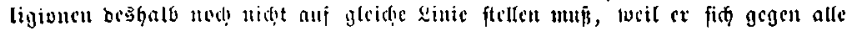

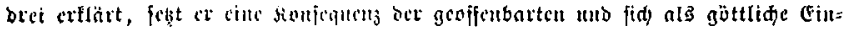

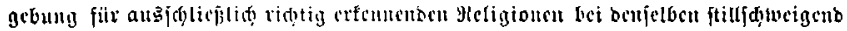

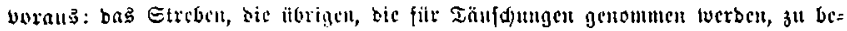

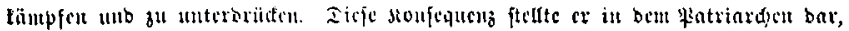

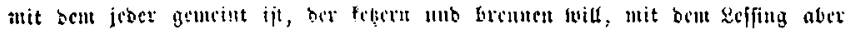

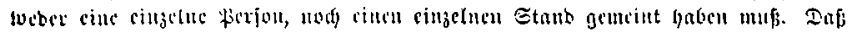

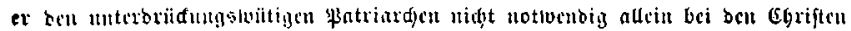

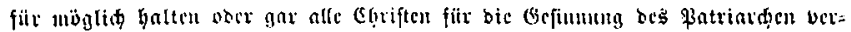

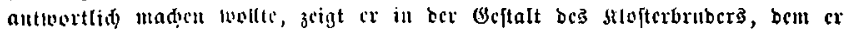

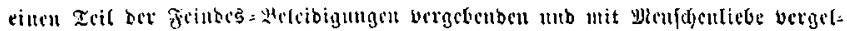

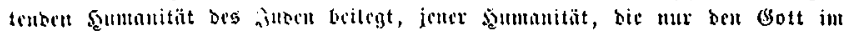

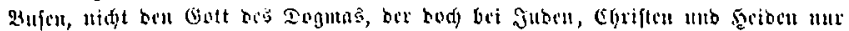

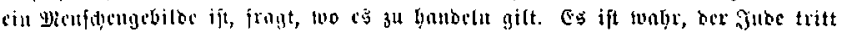

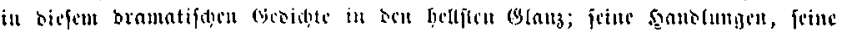

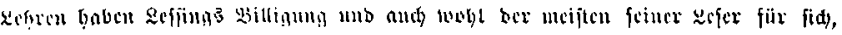

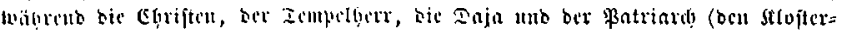

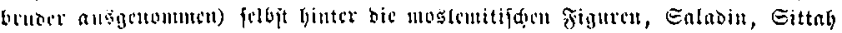

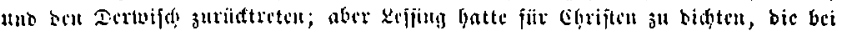

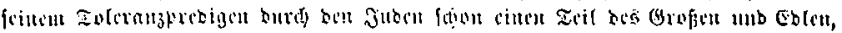

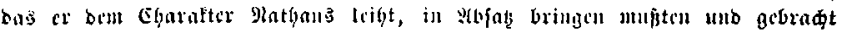

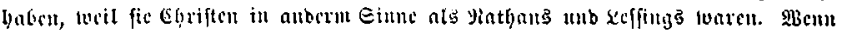

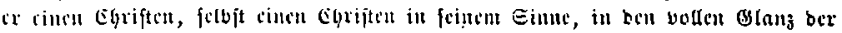

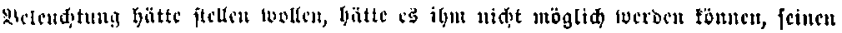

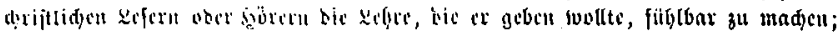

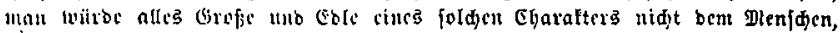

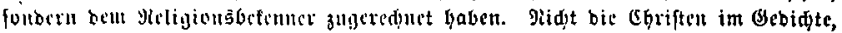

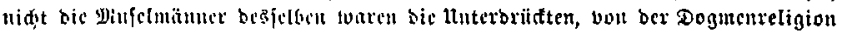

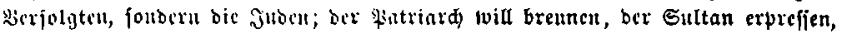

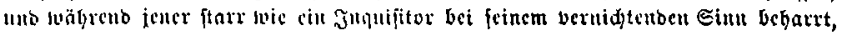

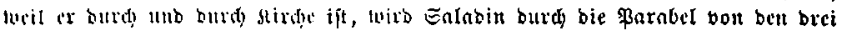

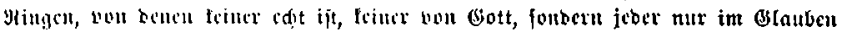

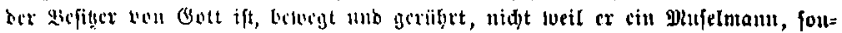

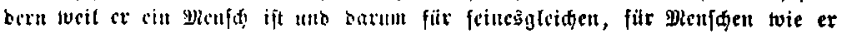

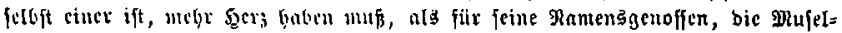

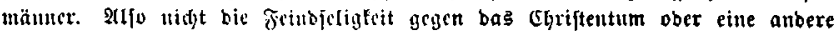

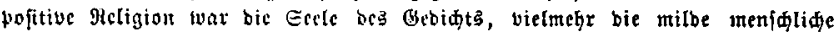
iiberjetgung whb Gabifbcit, baj in allen biefen olaubensformen ein einfader

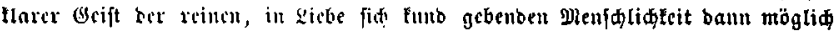

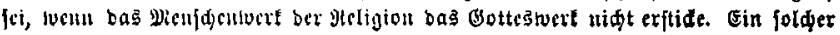


Geift werbe getommen feill, ivenu nady Iangen Iangen Jahren bie \$artsien wieber

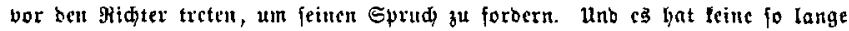

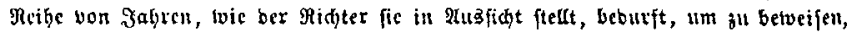

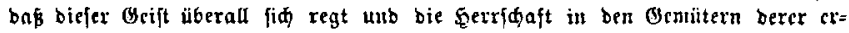

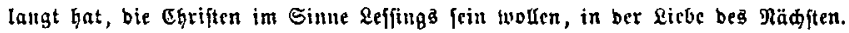
Eeffing Rathan bat bem Chriftentume mebr gemul, als aller Eifer allex Beloten affer Jahrounberte.

Qefifing meinte, jein Mathan werbe twoh! nie aufo Theater fommen. Tber fibon am 14. Mpril 1783 lourbe er ill Berlin, freilid ohue Teilnabme bes \$ubli=

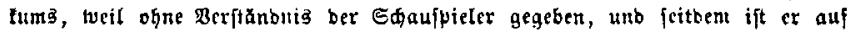
nUeu Bübnen, wenigfteng bes protefrantifder Deutidianba, beimif unb feine

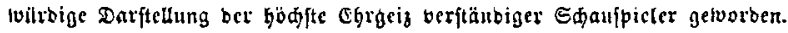

s. Hoebefe. 